Draft Version November 4, 2018

Preprint typeset using $\mathrm{LAT}_{\mathrm{E}} \mathrm{X}$ style emulateapj v. 08/22/09

\title{
FIRST RESULTS FROM THE SWARMS SURVEY. SDSS 1257+5428: A NEARBY, MASSIVE WHITE DWARF BINARY WITH A LIKELY NEUTRON STAR OR BLACK HOLE COMPANION.
}

\author{
Carles Badenes $^{1,2,3,4}$, Fergal Mullally ${ }^{1}$, Susan E. Thompson ${ }^{5}$ And Robert H. Lupton ${ }^{1}$ \\ Draft Version November 4, 2018
}

\begin{abstract}
We present the first results from the SWARMS survey, an ongoing project to identify compact white dwarf (WD) binaries in the spectroscopic catalog of the Sloan Digital Sky Survey. The first object identified by SWARMS, SDSS $1257+5428$, is a single-lined spectroscopic binary in a circular orbit with a period of $4.56 \mathrm{hr}$ and a semiamplitude of $322.7 \pm 6.3 \mathrm{~km} \mathrm{~s}^{-1}$. From the spectrum and photometry, we estimate a WD mass of $0.92_{-0.32}^{+0.28} \mathrm{M}_{\odot}$. Together with the orbital parameters of the binary, this implies that the unseen companion must be more massive than $1.62_{-0.25}^{+0.20} \mathrm{M}_{\odot}$, and is in all likelihood either a neutron star or a black hole. At an estimated distance of $48_{-19}^{+10} \mathrm{pc}$, this would be the closest known stellar remnant of a supernova explosion.

Subject headings: binaries:close, spectroscopic — white dwarfs — supernovae: general
\end{abstract}

\section{INTRODUCTION}

The vast majority of stars with masses below $8 \mathrm{M}_{\odot}$ end their lives as white dwarfs (WDs). Left to their own devices, WDs will just cool and fade away, but WDs that form part of close binary systems can interact with their companions, steering away from the normal course of isolated stellar evolution and giving rise to a plethora of astrophysical phenomena. When the companion of the WD is another compact object (a second WD, a neutron star $[\mathrm{NS}]$, or a black hole $[\mathrm{BH}]$ ), we refer to these systems as compact WD binaries, or CWDBs. The orbital evolution of detached CWDBs is governed by the emission of gravitational waves (GWs, Peters \& Mathews 1963; Paczyński 1967). For systems with small enough separations, loss of angular momentum leads to a merger of the two components in a time that is short enough to be of astrophysical interest. This kind of CWDBs constitute the largest population of Galactic GW sources (Evans et al. 1987), dominating the GW foreground for future missions like LISA (Nelemans 2009). They can also provide important constraints on the still poorly understood process of common-envelope evolution in binary systems (Nelemans \& Tout 2005).

But perhaps the most interesting aspect of CWDBs is the outcome of the merging process at the end of their binary evolution. For systems composed of two CO WDs whose combined mass is above the Chandrasekhar limit, the merger might trigger a thermonuclear runaway and lead to a Type Ia supernova (SN) explosion (Iben \& Tutukov 1984; Webbink 1984). This possibility,

\footnotetext{
${ }^{1}$ Department of Astrophysical Sciences, Princeton University. Peyton Hall, Ivy Lane, Princeton NJ 085441001; badenes@astro.princeton.edu, mullally@astro.princeton.edu, rhl@astro.princeton.edu

2 Chandra Fellow

${ }^{3}$ Benoziyo Center for Astrophysics, Faculty of Physics, Weizmann Institute of Science, 76100 Rehovot, Israel

${ }^{4}$ School of Physics and Astronomy, Tel-Aviv University, 69978 Tel-Aviv, Israel

${ }^{5}$ Department of Physics and Astronomy, University of Delaware, Newark, DE 19716; and Delaware Asteroseismic Research Center, Mt. Cuba Observatory, Greenville, DE 19807; sthomp@physics.udel.edu
}

often referred to as the double degenerate WD (DDWD) SN Ia progenitor scenario, is the only theoretical model that naturally explains the absence of $\mathrm{H}$ in the nebular spectra of Type Ia SN (see Leonard 2007, and references therein), and it has motivated a number of searches for suitable candidate systems. The most comprehensive of these searches, the SPY survey (Napiwotzki et al. 2001), examined the spectra of $\sim 1000$ WDs to look for radial velocity $(\mathrm{RV})$ shifts in the characteristic absorption lines. The total number of detached DDWDs found to date by SPY and other surveys is around 100 Napiwotzki et al. 2004), but the periods and mass estimates have only been published for 24 systems (Nelemans et al. 2005). So far, none of these systems clearly fulfills the requisites to be a SN Ia progenitor.

In this paper and in a companion publication (Mullally et al. 2009), we present the first results from SWARMS, the Sloan White dwArf Radial velocity data Mining Survey. The aim of SWARMS is to mine the WD catalog in the spectroscopic data base of the Sloan Digital Sky Survey (SDSS, York et al. 2000) in search of CWDBs. Our ultimate goal is to find the DDWD progenitors of Type Ia SNe, or at least put constraints on the rate of DD mergers in the Galactic disk that can be compared with measurements of the local Type Ia SN rate. This will allow us to assess the viability of the DDWD progenitor scenario for SN Ia. This paper is organized as follows. Our data mining strategy is briefly described and compared to the SPY survey in Section 2, using as an example the CWDB SDSS J125733.63+542850.5 (henceforth, SDSS $1257+5428$ ), the first object discovered by SWARMS. In Section 3, we present the follow-up observations of SDSS $1257+5428$ that we have conducted in order to determine its orbital and spectral parameters. In Section 4, we discuss the implications of our findings for the nature of the unseen companion of SDSS $1257+5428$. We summarize our conclusions and outline the future prospects for our survey in Section [5]

\section{SWARMS: MINING THE SDSS DATA BASE FOR COMPACT WD BINARIES}

\subsection{General Strategy}


The first version of the SDSS WD catalog was published by Kleinman et al. (2004). The most recent version, presented in Eisenstein et al. (2006) (henceforth, E06), corresponds to Data Release 4 of the SDSS (Adelman-McCarthy et al. 2006), and it is currently the largest catalog of its kind, with 9316 spectroscopically confirmed WDs. This number should be roughly doubled in the forthcoming DR7 version. Many publications have analyzed the SDSS spectra of WDs and related objects like subdwarf stars and cataclysmic variables (see E06 for a list of references), but so far all this work has focused on the final co-added spectra. All the SDSS spectra are in fact taken in three or more exposures, approximately 15 minutes long (Stoughton et al. 2002), in order to facilitate cosmic ray rejection. The exact number of exposures and the time lapsed between them varies from plate to plate, depending on the observing conditions at the time, but exposures are usually taken back to back during the same night. Starting with DR7 (Abazajian et al. 2009), the individual exposures are available separately, which opens the domain of time resolved spectroscopy in the SDSS data base.

The goal of SWARMS is to take advantage of this situation in the context of the WD spectra, looking for RV shifts among different exposures of the same object that may be the hallmark of a massive companion. To facilitate the search, we have started by considering only WDs with 'dominant type' DA or DB in the E06 catalog, i.e., objects that have a relatively simple spectrum with absorption lines from either $\mathrm{H}$ or He. From this list, we have discarded any objects with excess flux in the red part of the spectrum, which are usually classified as detached WD+M star binaries (Silvestri et al. 2006). This initial triage yields 7956 objects, which we have examined for RV shifts using several automated techniques supplemented by visual examination. A detailed description of our search strategy and the implications for completeness, the derivation of merger rates, and other related issues will be the subject of a forthcoming publication.

\subsection{SWARMS vs. SPY}

Before going on to discuss specific results, it is instructive to outline the fundamental differences between SWARMS and the SPY survey (Napiwotzki et al. 2001). At a very basic level, both surveys are complementary, because the SPY objects were examined for RV shifts from the VLT telescopes in Paranal (Chile) and have therefore mostly southern declinations, while SDSS data are taken from the Apache Point Observatory (APO) in New Mexico and have mostly northern declinations. The SPY survey achieved an excellent RV accuracy of $\sim 2 \mathrm{~km} \mathrm{~s}^{-1}$ by using the high-resolution UVES spectrograph $(R=18500)$, but the demands of high-resolution spectroscopy imposed a stringent magnitude cutoff of $B \leq 16.5$ on the SPY WDs, even for the large collecting area $(8.2 \mathrm{~m})$ of the VLT Kueyen telescope (Napiwotzki et al. 2001). By comparison, the resolution that can be achieved by SWARMS with the spectrographs on the SDSS $2.5 \mathrm{~m}$ telescope $(R=1800$, York et al. 2000) is much lower, around $170 \mathrm{~km} \mathrm{~s}^{-1}$. But RV resolution is not the most important factor to find potential DDWD SN Ia progenitors. The most interesting systems are those with relatively massive components, or tight orbits, or both, which are expected to have RV

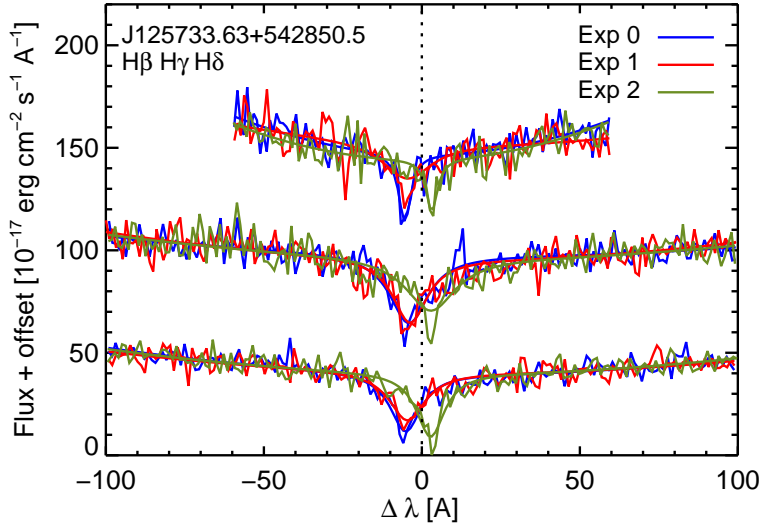

FIG. 1. - The three individual exposures of SDSS $1257+5428$ from the SDSS DR7 archive around the $\mathrm{H} \beta, \mathrm{H} \gamma$ and $\mathrm{H} \delta$ lines.

shifts higher than $\sim 150 \mathrm{~km} \mathrm{~s}^{-1}$ (Napiwotzki et al. 2004). Moreover, by foregoing the need for high-resolution spectroscopy we can examine dimmer WDs and hence many more objects. Through a combination of centroid fits and cross-correlation techniques, we have been able to achieve an accuracy of $\sim 120 \mathrm{~km} \mathrm{~s}^{-1}$, detecting RV shifts between consecutive SDSS exposures in a WD with a $g$ magnitude of 18.9 (Mullally et al. 2009). Thus, SWARMS can examine roughly an order of magnitude more objects than SPY, albeit at a much lower resolution. We can summarize our comparison by saying that SPY is better suited for a systematic study of the properties of DDWD systems, but SWARMS is better positioned to identify interesting objects, particularly the pre-mergers and SN Ia progenitors.

\subsection{The first $S W A R M S$ object: $S D S S 1257+5428$}

To illustrate the kind of objects that can be found by SWARMS, we present here results for SDSS $1257+5428$, the first CWDB identified by the survey. In the E06 catalog, SDSS $1257+5428$ was listed as a DA WD with a $g$ magnitude of 16.8 (see Table 1). There are three individual exposures of this WD in the DR7 SDSS data base. The WD is identified as a binary by the large shift in the centroid of the $\mathrm{H}$ lines between exposures 0 and 1 (taken on the night of October 32003 ) and exposure 2 (taken on the following night; see Figure 1). The shift has a value of $7.9 \AA$ at the $\mathrm{H}_{\beta}$ line, corresponding to a RV difference of $487 \mathrm{~km} \mathrm{~s}^{-1}$.

\section{FOLLOWUP OBSERVATIONS OF SDSS $1257+5428$}

\subsection{Data acquisition and reduction}

After a WD in the SDSS catalog is identified by SWARMS as a member of a binary system, follow-up observations are necessary to determine its period and amplitude, and obtain high signal-to-noise spectra that can be used to measure its effective temperature $T_{\text {eff }}$ and gravity $\log g$, calculate its mass, and constrain the nature of its companion. In the case of SDSS $1257+5428$, the follow-up observations were conducted with the Dual Imaging Spectrograph (DIS) at the $3.5 \mathrm{~m}$ ARC telescope at APO. A total of 23 spectra were taken on the nights of February 5, 6, and 14, 2009. We used the B1200 grating with a 1.5 " slit to achieve a $0.62 \AA$ dispersion and a FWHM $1.8 \AA$ resolution. The integration time was 10 minutes for all spectra, bracketed by exposures of $\mathrm{He}, \mathrm{Ne}$ 
TABLE 1

OBSERVATIONAL PROPERTIES OF SDSS $1257+5428$

\begin{tabular}{|c|c|}
\hline Parameter & Value \\
\hline \multicolumn{2}{|c|}{ Parameters from SDSS } \\
\hline $\begin{array}{l}R A(\mathrm{~J} 2000) \\
D e c(\mathrm{~J} 2000) \\
u(\mathrm{Mag}) \\
g(\mathrm{Mag}) \\
r(\mathrm{Mag}) \\
i(\mathrm{Mag}) \\
z(\mathrm{Mag}) \\
\text { Spectral type } \\
T_{\text {eff }}(\mathrm{K}) \\
\log (g)\end{array}$ & $\begin{array}{c}12 \mathrm{~h} 57 \mathrm{~m} 33.63 \mathrm{~s} \\
+54^{\circ} 28^{\prime} 50.5^{\prime \prime} \\
17.355 \pm 0.009^{\mathrm{a}} \\
16.844 \pm 0.004^{\mathrm{a}} \\
16.732 \pm 0.005^{\mathrm{a}} \\
16.722 \pm 0.005^{\mathrm{a}} \\
16.762 \pm 0.010^{\mathrm{a}} \\
\text { DA b } \\
8750 \pm 25^{\mathrm{b}} \\
9.0 \pm 0.005^{\mathrm{b}}\end{array}$ \\
\hline \multicolumn{2}{|c|}{ Orbital parameters (APO) } \\
\hline $\begin{array}{l}K_{A}\left(\mathrm{~km} \mathrm{~s}^{-1}\right) \\
P(\mathrm{hr}) \\
T_{0}(\mathrm{JD}) \\
\gamma_{A}\left(\mathrm{~km} \mathrm{~s}^{-1}\right) \\
\chi^{2}\end{array}$ & $\begin{array}{c}322.7 \pm 6.3 \\
4.5550 \pm 0.0007 \\
2454868.1182 \pm 0.0006 \\
-28.9 \pm 4.6 \\
44.4^{\mathrm{c}}\end{array}$ \\
\hline \multicolumn{2}{|c|}{ Spectral and Photometric Parameters (APO, SDSS) } \\
\hline $\begin{array}{l}T_{e f f}(\mathrm{~K}) \\
\log (g) \\
M_{A}\left(\mathrm{M}_{\odot}\right) \\
D(\mathrm{pc}) \\
t_{C o o l}(\mathrm{Gyr})\end{array}$ & $\begin{array}{c}\sim 9000^{\mathrm{d}} \\
\sim 8.5^{\mathrm{d}} \\
0.92_{-0.32}^{+0.28 \mathrm{e}} \\
48_{-19}^{+10 \mathrm{e}} \\
2.0 \pm 1.0^{\mathrm{e}}\end{array}$ \\
\hline \multicolumn{2}{|c|}{ Companion Parameters } \\
\hline $\begin{array}{l}M_{B} \sin (i)\left(\mathrm{M}_{\odot}\right) \\
M_{B} \text { for } i=60^{\circ}\left(\mathrm{M}_{\odot}\right) \\
t_{\text {Merge }} \text { for } i=90^{\circ}(\mathrm{Myr}) \\
t_{\text {Merge }} \text { for } i=60^{\circ}(\mathrm{Myr})\end{array}$ & $\begin{array}{c}1.62_{-0.25}^{+0.20} \\
2.10_{-0.30}^{+0.13} \\
511_{-141}^{+342} \\
267_{-70}^{+165}\end{array}$ \\
\hline $\begin{array}{l}\text { a DR7 values. } \\
\text { b Listed autofit results fr } \\
\text { c For } 23 \text { data points }(19 \mathrm{~d} \\
\text { d Spectral parameters from } \\
\text { tolerance ranges due to t } \\
\text { Section } 3.3 \\
\text { e See Section } 3.3 \text { for an exp } \\
\text { on } M_{A} \text { and } D \text {. }\end{array}$ & $\begin{array}{l}06 . \\
\text { APO fits are listed withou } \\
\text { w quality of the fits, se } \\
\text { ion of the tolerance range }\end{array}$ \\
\hline
\end{tabular}

and Ar arc lamps to ensure a proper wavelength calibration. Flux was calibrated each night by taking several exposures of the spectrophotometric standard Feige 67 (Oke 1990). The data were reduced using standard longslit IRAF routines (Tody 1993). A prominent Hg emission line at $4358 \AA$, courtesy of the inhabitants of White Sands, NM, confirmed that the wavelengths are calibrated to better than the instrumental resolution: the standard deviation of the line over 8 spectra (one night) is $0.1 \AA$. The final spectra span between 3770 and 5030 $\AA$, and clearly show all the lines in the Balmer series from $\mathrm{H}_{\beta}$ to $\mathrm{H}_{10}$.

\subsection{Orbit}

We measured the RV shift of each APO spectrum by fitting the centroid of the $\mathrm{H}_{\beta}$ line after applying the standard Solar System barycentric corrections. We used a Lorentzian profile with a Gaussian core (Thompson et al. 2004), adjusting the fit parameters with MPFIT, a version of the Levenberg-Marquardt algorithm adapted for IDL (Markwardt 2009). The resulting RV curve is well fit

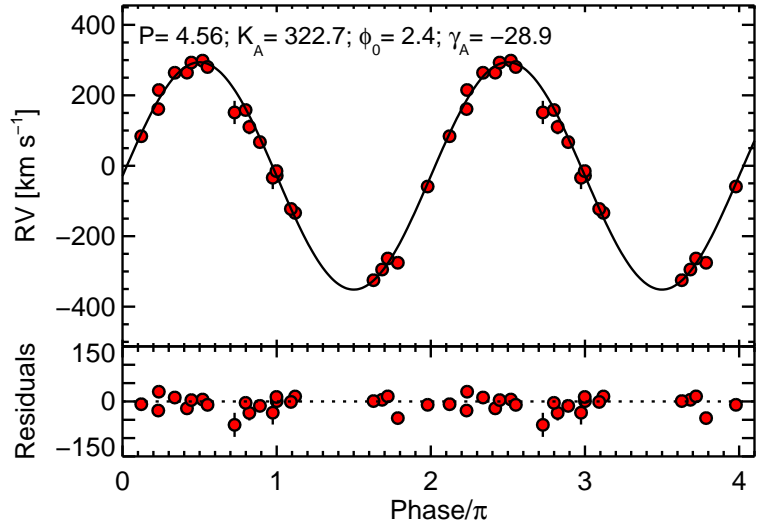

FIG. 2.- RV curve for SDSS $1257+5428$, folded in phase with the best-fit period.

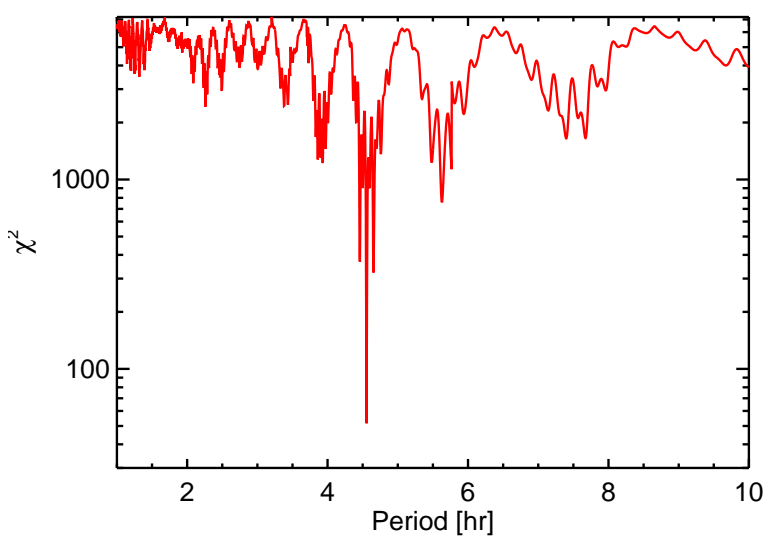

FIG. 3.- Goodness-of-fit parameter $\left(\chi^{2}\right)$ as a function of period for the RV curve of SDSS $1257+5428$.

by a circular orbit of the form $K_{A} \sin \left(\left(2 \pi\left(t-T_{0}\right) / P\right)\right)+$ $\gamma_{A}$, where $P$ is the period, $K_{A}$ the semiamplitude, $T_{0}$ a temporal offset marking the zero-point of the curve, and $\gamma_{A}$ a constant RV offset (see Figure 21). We found that the nine day baseline of the APO spectra strongly constrains the period of the binary to $P=4.5550 \pm 0.0007 \mathrm{hr}$, (Figure 3), with a semiamplitude of $K_{A}=322.7 \pm 6.3 \mathrm{~km} \mathrm{~s}^{-1}$ (see Table1 for a complete list of the orbital parameters).

\subsection{Spectrum \\ 3.3.1. Spectral fits}

The autofit results from $\mathrm{E} 06$ give $T_{\text {eff }}=8750 \pm 25 \mathrm{~K}$ and $\log g=9.0 \pm 0.005$ for SDSS $1257+5428$, but these parameters are derived from the co-added DR4 SDSS spectrum of an object that was not known to be a shortperiod binary. In order to make the best possible determination of $T_{\text {eff }}$ and $\log g$, we have co-added the 23 APO spectra after removing the RV shifts. The resulting spectrum has a $\mathrm{S} / \mathrm{N}$ of 160 , much higher than the co-added SDSS spectrum ( $\mathrm{S} / \mathrm{N}=30)$.

We have used the MPFIT routine to fit the co-added APO spectrum with the latest (June 2008) version of the Koester atmosphere models descrbed in Finley et al. (1997), adjusted with a first order polynomial. This spectral fitting has proved a challenging endeavor. The raw (unreduced) $\chi^{2}$ parameter has two distinct minima in the $T_{e f f}, \log g$ plane: one around $T_{\text {eff }}=35000 \mathrm{~K}$, $\log g=7.5$ and one around $T_{\text {eff }}=8500 \mathrm{~K}, \log g=8.5$ (see Figure 4, left panel). The hot solutions provide 

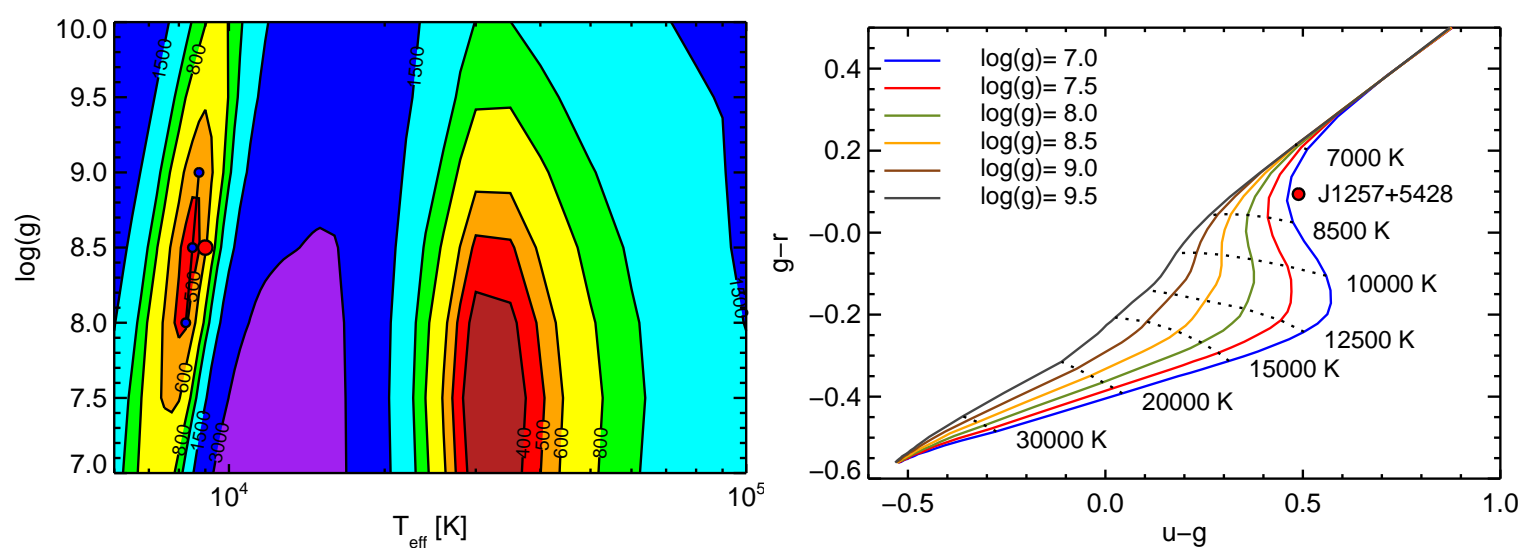

FIG. 4.- Constraints on $T_{\text {eff }}$ and $\log g$ for SDSS 1257+5428. Left: surface contours of the raw $\chi^{2}$ parameter obtained from fitting the co-added APO spectrum of SDSS $1257+5428$ to the most recent version of the models by Finlev et al. (1997). The best-fit solution $\left(T_{\text {eff }}=9000 \mathrm{~K}, \log g=8.5\right.$, see text and Figure 5) is indicated by a large red circle. The models used to derive the confidence ranges for the WD mass are indicated by smaller blue circles, joined by straight lines. Right: comparison between the DR7 SDSS photometry of SDSS $1257+5428$ (red circle) and the photometric models of Holberg \& Bergeron (2006). The cooling tracks are labeled by log $g$, with points at the same $T_{\text {eff }}$ represented by dotted lines.

slightly lower $\chi^{2}$ values than the cold solutions, but neither fits are very impressive (see Figure 5 for the bestfit cold solution - the visual quality of the best-fit hot solution is similar). This degeneracy between cold and hot spectral solutions is common in DA WDs, and it can usually be broken by including photometric information (Kleinman et al. 2004). In the right panel of Figure 4. we compare the DR7 SDSS photometry of SDSS $1257+5428$ (Table 11) with the photometric models of Holberg \& Bergeron (2006). The hot solutions can be discarded with a high degree of confidence. The cold solutions come very close to reproducing the SDSS photometry, with the models along the cold $\chi^{2}$ minimum being offset from the data by only $\sim-0.15$ magnitudes in $u-g$. This kind of discrepancy is not uncommon in comparisons between the SDSS photometry of DA WDs and cooling models (Holberg \& Bergeron 2006, see also Section 3.3 .2 below). Including the full reddening correction of Schlegel et al. (1998) changes the $u-g$ color by -0.025 magnitudes and the $g-r$ color by -0.018 magnitudes, which reduces the discrepancy by a small amount.

Even though it is not exactly at the bottom of the local $\chi^{2}$ minimum, we have found that the spectral model with $T_{\text {eff }}=9000 \mathrm{~K}$ and $\log g=8.5$ (Figure 5) provides the best match to the continuum and the cores of the high order Balmer lines, which should be more sensitive to the value of $\log g$ (Finlev et al. 1997). We consider this model our 'best fit', although it cannot be said that the spectral adjustment shown in Figure 5 is particularly good. Nevertheless, the values of $T_{\text {eff }}$ and $\log g$ that we obtain are reasonably close to those found by E06 using the co-added SDSS spectrum. This agreement between different analysis techniques applied to different data sets of the same object is encouraging, and supports our choice of spectral model.

\subsubsection{Why are the fits so poor?}

The failure of theoretical models to reproduce the details of the spectrum and photometry of SDSS $1257+5428$ is not entirely unexpected. Even the most updated versions of the Koester atmosphere models have problems for WDs with $T_{\text {eff }}<12000 \mathrm{~K}$. At these tem- peratures, the contribution of neutral and charged perturbers to the equation of state is of the same order of magnitude, and the size of the convective envelope of the WD has an abrupt increase (Koester et al. 2008). These effects have been put forward as possible causes for the observed increase of the average effective gravity for WDs in this temperature range (Kepler et al. 2007; Bergeron et al. 2007). The discrepancies shown in Figure 5. however, are rather large, and they should not be written off as shortcomings of the models before considering alternative explanations.

The high $\mathrm{S} / \mathrm{N}$ of our co-added APO spectrum reveals some unusual features in the continuum around $4600 \AA$ and $4750 \AA$. The features are not present in the photometric standard Feige 67, which indicates that the flux calibration is not at fault. None of the calibration frames, flat fields or biases have flaws that could have caused these features. Other WDs that we observed with the same spectrograph in the same mode (Mullally et al. 2009) do not show them, but these are fainter objects with worse $\mathrm{S} / \mathrm{N}$ in the co-added spectra. At this stage, we have no explanation for the presence of these localized features, but they are clearly not large enough to influence the mismatch between the models and the observed spectrum.

It is possible that the spectrum of SDSS $1257+5428$ is simply distorted by optical emission from its binary companion. The co-added SDSS spectrum shows no traces of line emission or a substantial excess in the red spectrograph, clear signs of the presence of a nondegenerate companion that would have prompted a DA+M classification for SDSS $1257+5428$ by E06 (this is ruled out by the RV curve as well, see the discussion in Section 4). A WD companion is more difficult to discard from the point of view of the spectrum alone. We have examined the individual APO spectra that are closer to quadrature, when the relative RV shift of the individual components should be larger, but found no sign of artifacts or asymmetries in the Balmer lines that might indicate the presence of a second DA WD of similar temperature. A substantially hotter WD of any spectral class would rapidly overwhelm the spectrum of SDSS $1257+5428$, having a large impact on the SDSS photometry shown in Figure 

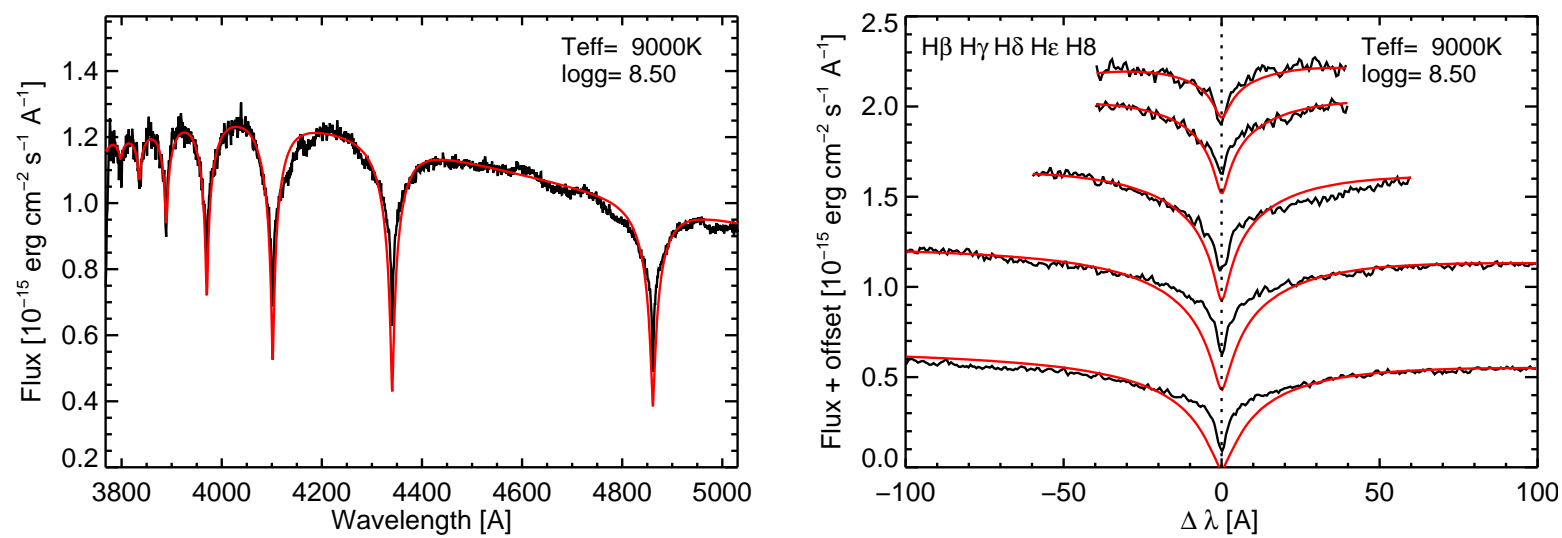

FIG. 5.- Best-fit spectral solution for SDSS 1257+5428: $T_{\text {eff }}=9000 \mathrm{~K}, \log g=8.5$.

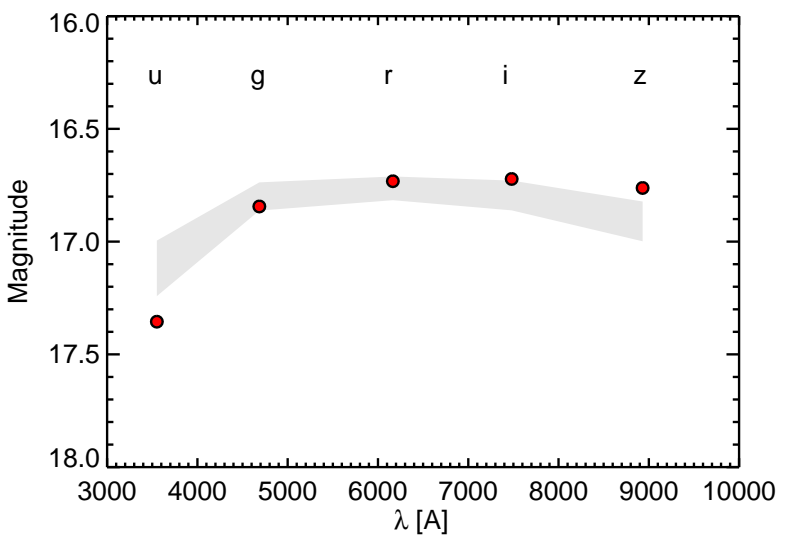

FIG. 6.- Comparison between the SED of SDSS $1257+5428$ from the five-band DR7 SDSS photometry (red circles) with the predictions of Holberg \& Bergeron (2006) for WD models along the cold $\chi^{2}$ minimum, $T_{\text {eff }}$ between 8000 and $9000 \mathrm{~K}$ and $\log g$ between 8.0 and 9.0 (shaded area).

4. The only possibility left is a non-DA WD of similar or lower temperature with a rather featureless spectrum. In Figure 6 we display the spectral energy density (SED) of SDSS $1257+5428$ together with the range covered by the models of Holberg \& Bergeron (2006). The SEDs of models along the cold $\chi^{2}$ minimum are roughly consistent with the five-band photometry of SDSS $1257+5428$. There might be a hint of an excess in the $z$ band, but infrared data would be required to confirm this possibility. On the other hand, all the models seem to overpredict the flux in the $u$ band, an effect that was already apparent in the color-color plot shown in Figure 4. Since this is a flux deficit instead of an excess, it is hard to explain by invoking the presence of a companion. In fact, Holberg \& Bergeron (2006) find similar problems in the $u$ band for other cold DA WDs (see their Figure 6).

We conclude that the shortcomings of the WD models in this temperature range are indeed the most likely explanation for the poor quality of the spectral fit shown in Figure 5. A cold WD companion cannot be ruled out based on the spectral information alone, but neither is there any evidence in the observations that requires the presence of such a companion. We shall revisit this possibility in Section 4

\subsubsection{Estimating the mass of the primary WD}

The issues with the models that we have discussed require that we be extremely careful with the interpretation of the spectral fits. In particular, we cannot justify the calculation of confidence ranges around the fitted parameters in the usual way (i.e., based on the $\chi^{2}$ statistic). But we are not interested in the confidence ranges around $T_{\text {eff }}$ and $\log g$ per se. Rather, our goal is to use the spectrum of SDSS $1257+5428$ to measure its mass and constrain the nature of its unseen companion.

An estimate for the mass of SDSS $1257+5428$ can be obtained by examining the performance of the spectral models around the cold minimum in $\chi^{2}$ space. The cooling curves of Fontaine et al. (2001) included in the models of Holberg \& Bergeron (2006) give a value of $M_{A}=0.92 \mathrm{M}_{\odot}$ for our best-fit spectral solution, which is probably a good initial guess for the mass of SDSS $1257+5428$. Along the cold $\chi^{2}$ minimum, the spectral solutions with $T_{\text {eff }}=8250,8500$, and $8750 \mathrm{~K}$; and $\log g=8.0,8.5$, and 9.0 (indicated by the small blue circles in the left panel of Figure 44) appear to bracket the effective gravity values that are in best agreement with the higher order Balmer lines in the spectrum, as shown in Figure 7 Any models with $\log g$ outside this range result in extremely poor fits and must be discarded, including the models with lower $T_{\text {eff }}$ and lower $\log g$ that seem to be preferred by the photometry (right panel of Figure 4). Since we have already ruled out a DA WD companion of similar temperature, we expect the shape of the higher order Balmer lines to be a reliable probe of the mass of SDSS $1257+5428$, even if the underlying continuum is somewhat distorted.

The spectral models shown in Figure 7 translate into masses between 0.60 and $1.20 \mathrm{M}_{\odot}$, which we will adopt as conservative lower and upper limits to $M_{A}$. It is unfortunate that the poor performance of the spectral models in this temperature range does not allow for a more accurate measurement of $M_{A}$, but we believe that our estimate is reasonable, and probably the best that can be done with current tools. We note that the models we contemplate here span a range of $\log g$ values around the best-fit solution $( \pm 0.5)$ that is comfortably larger than the reported increase for $T_{\text {eff }}<12000 \mathrm{~K}(\sim 0.2$, Kepler et al. 2007; Bergeron et al.|2007). Also, our lower limit to $M_{A}$ is close to the mean mass of DA WDs with $T_{\text {eff }} \geq 12000 \mathrm{~K}\left(0.58 \mathrm{M}_{\odot}\right.$, Kepler et al. 2007), which would be the best guess for the mass of a WD in absence of any spectral or photometric information. For 


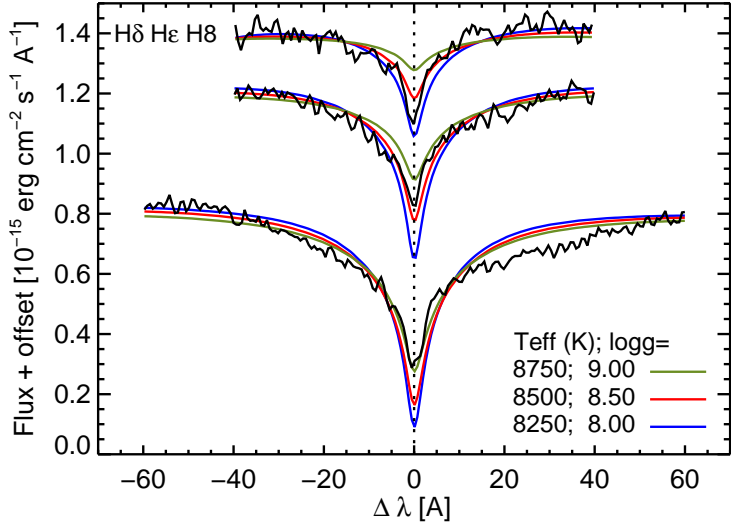

FIG. 7.- Comparison between the high order Balmer lines $\left(\mathrm{H}_{\delta}\right.$, $\mathrm{H}_{\epsilon}$, and $\mathrm{H}_{8}$ ) in the spectrum of SDSS $1257+5428$ and the three models along the cold $\chi^{2}$ minimum indicated with small blue circles in the left panel of Figure 4 Although higher order lines $\left(\mathrm{H}_{9}\right.$ and $\mathrm{H}_{10}$ ) are present in the spectrum, the $\mathrm{S} / \mathrm{N}$ is too low for a meaningful comparison with the models.

all these reasons, we are confident that the true mass of SDSS $1257+5428$ lies within the range of values that we propose here. For comparison purposes, the best-fit hot spectral model $\left(T_{e f f}=35000 \mathrm{~K}, \log g=7.5\right)$ translates into a value of $0.45 \mathrm{M}_{\odot}$ for $M_{A}$. With the set of limiting spectral models around the best-fit cold solution shown in Figure 4, the Holberg \& Bergeron curves yield a cooling age of $2.0 \pm 1.0$ Gyr. The absolute $g$ magnitude is $13.4_{-0.4}^{+1.1}$, which results in a distance of $D=48_{-19}^{+10} \mathrm{pc}$ for SDSS $1257+5428$.

\section{THE COMPANION OF SDSS 1257+5428: NEUTRON} STAR OR BLACK HOLE?

Combining our estimate for the mass of SDSS $1257+5428\left(M_{A}=0.92_{-0.32}^{+0.28} \mathrm{M}_{\odot}\right)$ with the values of $P$ $(4.5550 \pm 0.0007 \mathrm{hr})$ and $K_{A}\left(322.7 \pm 6.3 \mathrm{~km} \mathrm{~s}^{-1}\right)$ derived in Section 3.2. we obtain $M_{B} \sin (i)=1.62_{-0.25}^{+0.20} \mathrm{M}_{\odot}$ for its unseen companion, with $M_{B}$ and the inclination angle $i$ being degenerate as in all single-lined binaries. A plot of the companion mass as a function of $\cos (i)$ is shown in Figure 8 . We stress that the properties of the RV curve alone require a massive companion, regardless of the estimated value for $M_{A}: M_{B}$ must be more massive than $0.66 \mathrm{M}_{\odot}$ even for $M_{A}=0$ (i.e., assuming the value of $M_{A}$ is negligible for the dynamics of the system), and more massive than $1.08 \mathrm{M}_{\odot}$ for $M_{A}=0.3 \mathrm{M}_{\odot}$. With any reasonable range of masses for the primary, a nondegenerate stellar companion would have a spectral type $G$ or earlier, which is clearly incompatible with the observations of SDSS $1257+5428$. The companion must therefore be a compact object, either another WD, a NS or a $\mathrm{BH}$.

Could the companion be a WD? We have not been able to discard this possibility from the point of view of the optical spectrum alone, but the properties of the RV curve make it very unlikely. The companion could only be a WD if our mass estimate for $M_{A}$ was completely off, and even then, it would have to be a very massive object (above $1.0 \mathrm{M}_{\odot}$ for any reasonable value of $M_{A}$ ), cool object, without any prominent absorption lines (see discussion in Section 3.3.2). Such massive WDs are extremely rare. But we have found no evidence to indicate that the shape of the Balmer lines in the spectrum of SDSS $1257+5428$ is distorted in any way, so there is no

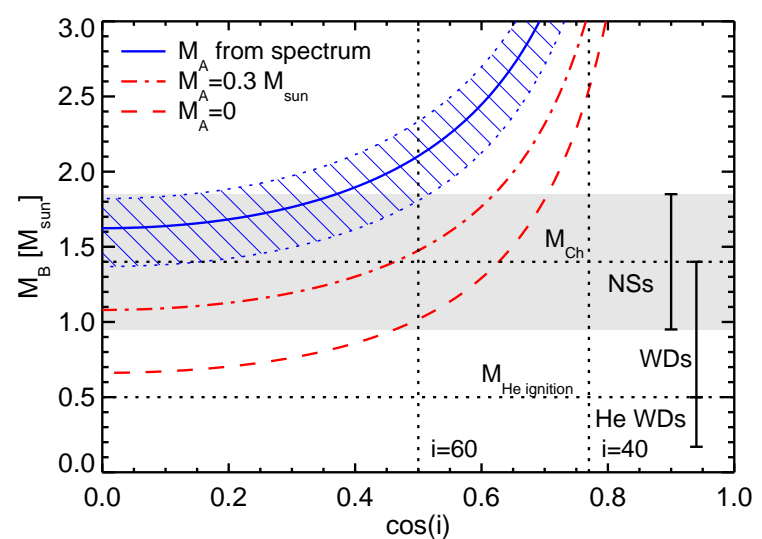

FIG. 8. - Mass function for the companion of SDSS $1257+5428$. The solid blue curve represents the values obtained using the best estimate for the WD mass $\left(M_{A}=0.92 \mathrm{M}_{\odot}\right)$, and the striped region is bound by the upper and lower limits to the WD mass obtained from the spectrum $\left(M_{A}=0.60\right.$ and $\left.1.20 \mathrm{M}_{\odot}\right)$. For comparison, the mass functions for $M_{A}=0.3 \mathrm{M}_{\odot}$ and $M_{A}=0$ are also shown (dash-dotted and dashed red plots). The range of masses for known NSs from Lattimer \& Prakash (2007) has been highlighted with a shaded region, taking into account the recent re-evaluation of the mass of PSR $0751+1807$ by Nice et al. (2008).

reason to doubt our conservative estimate for $M_{A}$. If this estimate holds, assuming the smallest possible value of $M_{A}\left(0.6 \mathrm{M}_{\odot}\right)$, the orbit would have to be nearly edge-on $\left(i \geq 82^{\circ}\right)$ for $M_{B}$ to be below the Chandrasekhar limit $M_{C h}$. This inclination is expected to happen randomly in only $14 \%$ of binary systems, but SWARMS does have a strong observational bias towards finding high inclination systems. Even then, the companion would be more massive than the largest known WDs (1.33 to $1.35 \mathrm{M}_{\odot}$, see Kepler et al. 2007; Barstow et al. 1995). In this unlikely circumstance, the SDSS $1257+5428$ system would have a combined mass at least $43 \%$ larger than $M_{C h}$, but might not be a SN Ia progenitor if the companion turns out to be an ONe WD instead of a CO WD (Garcia-Berro et al. 1997). Considering all the evidence, the most likely possibility by far is that the companion of SDSS $1257+5428$ be the stellar remnant of a supernova explosion, either a $\mathrm{NS}$ or a $\mathrm{BH}$. We note that our estimated distance range places this object closer to the Solar System than any other known NS (Posselt et al. 2007).

The puzzle of the nature of the companion of SDSS $1257+5428$ cannot be solved with the observations that we present in this paper, and must be the subject of future work. It is, however, interesting to speculate about the possibilities. For our best-fit value of $M_{A}\left(0.92 \mathrm{M}_{\odot}\right)$, $M_{B}$ is below $1.86 \mathrm{M}_{\odot}$ (the largest masured mass for a NS, see Lattimer \& Prakash 2007; (Nice et al. 2008) for $i \geq 67^{\circ}$, which has a random likelihood of $39 \%$. In this case, the mass of the WD and the circularity of the orbit would place the system in the class of 'intermediate-mass' WD+NS binaries (see Table 1 in Stairs 2004). Current models for binary stellar evolution predict that these systems undergo unstable mass transfer during a commonenvelope phase (Stairs 2004), which results in the NS becoming a mildly recycled millisecond pulsar (MSP). This scenario would explain the high inferred mass for the companion of SDSS $1257+5428$, well above the average for a NS, as a byproduct of the mass transfer process (Lattimer \& Prakash 2007). If the NS is indeed an MSP, we expect the magnetic field to be low $\left(\sim 10^{9}\right.$ Gauss $)$, 
the pulsar lifetime to be large ( $\sim 1 \mathrm{Gyr})$, and the opening angle to be wide (possibly many tens of degrees, see Phinney \& Kulkarni 1994). Under these conditions, the prospects for detecting such a nearby MSP are good, although we note that the cooling time for the WD is also of the order of Gyr (see Section 3.3), and the MSP might have lost a large part of its magnetic field. For $M_{A}=0.92 \mathrm{M}_{\odot}$ and inclination angles below $67^{\circ}(61 \%$ random likelihood), the companion would probably be a stellar mass BH. In this case, we would not expect any kind of direct emission from it.

No radio or X-ray source appears at this location in any of the indexed astronomical catalogs, but this does not preclude the existence of a faint counterpart to the companion of SDSS $1257+5428$. This part of the sky has only been shallowly surveyed for MSPs in the radio. The most stringent limits are probably from the Green Bank $140 \mathrm{ft} 350 \mathrm{MHz}$ survey, which had a nominal sensitivity of 12-15 mJy (Saver et al. 1997). In the Xrays, this location has never been observed with Chandra or $X M M-N e w t o n$, and a faint nearby source could have easily escaped detection by the ROSAT All-Sky Survey. A systematic cross-correlation between stellar sources in SDSS (including all the WDs from E06) and the ROSAT catalogues was performed by Agüeros et al. (2009), who found no counterpart to SDSS $1257+5428$.

One interesting implication of the companion of SDSS $1257+5428$ being a NS or BH is that the system should have received some kind of kick from the SN explosion. In principle, the spatial velocity of SDSS $1257+5428$ can be determined by the temporal average of the nongravitational Doppler shifts in the spectrum, which gives the radial component, and the proper motion measured

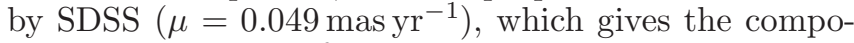
nent on the plane of the sky. Taking our distance estimate, the proper motion translates into a transverse velocity of $11_{-4}^{+2} \mathrm{~km} \mathrm{~s}^{-1}$. The radial component is more difficult to estimate, because the constant component to the RV curve $\left(\gamma_{A}=-28.9 \pm 4.6 \mathrm{~km} \mathrm{~s}^{-1}\right.$ in our fit, see Section 3.2 is the combination of the true radial velocity and the gravitational redshift of the WD. The value of the gravitational redshift depends on the WD mass, which we cannot measure with accuracy (see Section 3.3), and the WD radius, which is also model dependent. A detailed estimate of the gravitational redshift for SDSS $1257+5428$ is outside the scope of this work, but for a massive $\sim 1 \mathrm{M}_{\odot}$ WD, we expect it to be of the order of $90 \mathrm{~km} \mathrm{~s}^{-1}$ (Wegner \& Reid 1991), which would require a radial velocity around $-120 \mathrm{~km} \mathrm{~s}^{-1}$. The total spatial velocity would then be $\sim 120 \mathrm{kms}^{-1}$, mostly in the radial direction, which is comparable to the measured kicks for pulsars in binary systems (Wang et al. 2006).

Regardless of what the nature of the companion to SDSS $1257+5428$ turns out to be, the system is clearly very interesting from an astrophysical point of view, and new observations should yield exciting results in the near future. From the measured orbital parameters, the separation of the components must be small, with values of the semimajor axis larger than 0.02 AU only for $i \leq 17^{\circ}$ (0.0093 AU at $\left.i=60^{\circ}\right)$. If pulsations from a NS companion were detected, this could allow for a significant measurement of the Shapiro delay, as in PSR J0437-4715 (van Straten et al. 2001). The merging time $t_{M e r g e}$ of the SDSS $1257+5428$ system due to GW radiation is $\leq 511_{-141}^{+342} \mathrm{Myr}$, with the uncertainty in this upper limit arising from the uncertainty in the determination of $M_{A}$. For the canonical inclination angle $i=60^{\circ}, t_{\text {Merge }}$ becomes $267_{-70}^{+165} \mathrm{Myr}$. We note that only three WD+NS binaries with $t_{\text {Merae }}<t_{\text {Hubble }}$ were known previously (Stairs 2004; Kim et al. 2004), and SDSS 1257+5428 has a shorter period and $t_{\text {Merge }}$ than any of them. The discovery of a fourth object in this class should prompt a revision of the estimated $\mathrm{WD}+\mathrm{NS}$ merger rates and their expected contribution to the GW background (Kim et al. 2004).

The ultimate fate of SDSS $1257+5428$ is unclear. King et al. (2007) have proposed the merging of massive WDs onto NSs as a scenario for the origin of long-duration gamma-ray bursts without an accompanying SN like GRB 060614 (Gal-Yam et al. 2006; Della Valle et al. 2006), but theoretical simulations for the final accretion phase in this kind of events have not been performed yet. The coalescence of a $\mathrm{BH}$ with a massive WD may also lead to a gamma-ray burst (Fryer et al. 1999), but the observational signature depends again on the details of the final accretion phase, which are poorly understood. More exotic astrophysical transients, such as may be observed by present (PTF, Rau et al. 2009) and forthcoming (e.g. LSST, Ivezic et al. 2008) synoptic surveys, cannot be discarded as outcomes.

\section{CONCLUSIONS}

In this paper and a companion publication (Mullally et al. 2009) we have presented the first results from the SWARMS survey, an ongoing project aimed at discovering and characterizing CWDBs in general and DDWD SN Ia progenitors in particular among the WDs in the spectroscopic SDSS data base. Further results and a complete description of the survey will be the subject of forthcoming publications. Our ultimate goal is to estimate a rate for the merger of DDWD SN Ia progenitors in the Galaxy, and to use that rate, in combination with the results from SPY and other surveys, to assess the viability of the DDWD progenitor scenario for Type Ia SNe.

To demonstrate the capabilities of SWARMS, we have focused on SDSS $1257+5428$, the first object found by the survey. This WD was identified as a member of a binary system from the SDSS spectra, and follow-up observations were performed to measure its orbital and spectral parameters. The RV curve has a period of $4.5550 \pm 0.0007$ hr, with a semiamplitude of $322.7 \pm 6.3 \mathrm{~km} \mathrm{~s}^{-1}$. The primary WD is cold $(\sim 9000 \mathrm{~K})$, which makes the spectral analysis somewhat challenging, but we have derived a conservative estimate of $0.92_{-0.32}^{+0.28} \mathrm{M}_{\odot}$ for its mass. This implies that the unseen companion must be larger than $1.62_{-0.25}^{+0.20} \mathrm{M}_{\odot}$, and is probably a NS or a $\mathrm{BH}$. At a distance of $D=48_{-19}^{+10} \mathrm{pc}$, this would be the closest remnant of a SN explosion to the Solar System. We have discussed the implications of our discovery, and suggested future avenues of research on this object. This kind of massive, close binary is a perfect example of the objects that can be found by SWARMS and other similar surveys like MUCHFUSS (Tillich et al. 2009). We anticipate more such discoveries, which we will report in the literature. We also plan to set up an on-line database of SWARMS 
objects.

We are grateful to the APO staff for assistance during our observations, to Alexei Filippenko for hosting CB at Berkeley during the initial stages of this project, and to the referee, Ralf Napiwotzki, for several comments that improved the quality of our manuscript. We also wish to thank Detlev Koester, who kindly provided an updated version of his grid of WD models and contributed useful suggestions. We acknowledge fruitful discussions with Lars Bildsten, Jenny Greene, Gijs Nelemans, Roman
Rafikov, Scott Ransom, Anatoly Spitkovsky, and Michael Strauss. At Princeton University, CB is supported by NASA through Chandra Postdoctoral Fellowship Award Number PF6-70046 issued by the Chandra X-ray Observatory Center, which is operated by the Smithsonian Astrophysical Observatory for and on behalf of NASA under contract NAS8-03060. CB also thanks the Benoziyo Center for Astrophysics for support at the Weizmann Institute of Science. SET thanks the Crystal Trust for their financial support.

\section{REFERENCES}

Abazajian, K. N., et al. 2009, ApJS, 182, 543

Adelman-McCarthy, J. K., et al. 2006, ApJS, 162, 38

Agüeros, M. A., Anderson, S. F., Covey, K. R., Hawley, S. L., Margon, B., Newsom, E. R., Posselt, B., Silvestri, N. M., Szkody, P., \& Voges, W. 2009, ApJS, 181, 444

Barstow, M. A., Jordan, S., O'Donoghue, D., Burleigh, M. R., Napiwotzki, R., \& Harrop-Allin, M. K. 1995, MNRAS, 277, 971

Bergeron, P., Gianninas, A., \& Boudreault, S. 2007, in Astronomical Society of the Pacific Conference Series, Vol. 372, 15th European Workshop on White Dwarfs, ed. R. Napiwotzki \& M. R. Burleigh, 29

Della Valle, M., et al. 2006, Nature, 444, 1050

Eisenstein, D. J., et al. 2006, ApJS, 167, 40 [E06]

Evans, C. R., Iben, I. J., \& Smarr, L. 1987, ApJ, 323, 129

Finley, D. S., Koester, D., \& Basri, G. 1997, ApJ, 488, 375

Fontaine, G., Brassard, P., \& Bergeron, P. 2001, PASP, 113, 409

Fryer, C. L., Woosley, S. E., Herant, M., \& Davies, M. B. 1999, ApJ, 520, 650

Gal-Yam, A., et al. 2006, Nature, 444, 1053

Garcia-Berro, E., Ritossa, C., \& Iben, I. J. 1997, ApJ, 485, 765

Holberg, J. B. \& Bergeron, P. 2006, AJ, 132, 1221

Iben, I. \& Tutukov, A. 1984, ApJS, 54, 335

Ivezic, Z., et al. 2008, arXiv:0805.2366

Kepler, S. O., Kleinman, S. J., Nitta, A., Koester, D. Castanheira, B. G., Giovannini, O., Costa, A. F. M., \& Althaus, L. 2007, MNRAS, 375, 1315

Kim, C., Kalogera, V., Lorimer, D. R., \& White, T. 2004, ApJ, 616,1109

King, A., Olsson, E., \& Davies, M. B. 2007, MNRAS, 374, L34

Kleinman, S. J., et al. 2004, ApJ, 607, 426

Koester, D., Kepler, S. O., Kleinman, S. J., \& Nitta, A. 2008, Journal of Physics: Conference Series, 172, 012006.

Lattimer, J. M. \& Prakash, M. 2007, Phys. Rep., 442, 109

Leonard, D. C. 2007, ApJ, 670, 1275

Markwardt, C. B. 2009, arXiv:0902.2850

Mullally, F., Badenes, C., \& Thompson, S. E. 2009, ApJ, submitted

Napiwotzki, R., et al. 2001, Astronomische Nachrichten, 322, 411

Napiwotzki, R., et al. 2004, in Astronomical Society of the Pacific Conference Series, Vol. 318, Spectroscopically and Spatially Resolving the Components of the Close Binary Stars, ed. R. W. Hilditch, H. Hensberge, \& K. Pavlovski, 402-410
Nelemans, G. 2009, Classical and Quantum Gravity, 26, 094030 Nelemans, G., Napiwotzki, R., Karl, C., Marsh, T. R., Voss, B., Roelofs, G., Izzard, R. G., Montgomery, M., Reerink, T., Christlieb, N., \& Reimers, D. 2005, A\&A, 440, 1087

Nelemans, G. \& Tout, C. A. 2005, MNRAS, 356, 753

Nice, D. J., Stairs, I. H., \& Kasian, L. E. 2008, in American Institute of Physics Conference Series, Vol. 983, 40 Years of Pulsars: Millisecond Pulsars, Magnetars and More, ed.

C. Bassa, Z. Wang, A. Cumming, \& V. M. Kaspi, 453-458

Oke, J. B. 1990, AJ, 99, 1621

Paczyński, B. 1967, Acta Astronomica, 17, 287

Peters, P. C. \& Mathews, J. 1963, Physical Review, 131, 435

Phinney, E. S. \& Kulkarni, S. R. 1994, ARA\&A, 32, 591

Posselt, B., Popov, S. B., Haberl, F., Trümper, J., Turolla, R., \& Neuhäuser, R. 2007, Ap\&SS, 308, 171

Rau, A., et al. 2009, arXiv:0906.5355

Sayer, R. W., Nice, D. J., \& Taylor, J. H. 1997, ApJ, 474, 426

Schlegel, D. J., Finkbeiner, D. P., \& Davis, M. 1998, ApJ, 500, 525

Silvestri, N. M., et al. 2006, AJ, 131, 1674

Stairs, I. H. 2004, Science, 304, 547

Stoughton, C., et al. 2002, AJ, 123, 485

Thompson, S. E., Clemens, J. C., van Kerkwijk, M. H., O'Brien, M. S., \& Koester, D. 2004, ApJ, 610, 1001

Tillich, A., Geier, S., Heber, U., Hirsch, H., Maxted, P., Gaensicke, B., Marsh, T., Napiwotzki, R., Østensen, R. \& Copperwheat, C. 2009, Journal of Physics: Conference Series, 172,012009

Tody, D. 1993, in Astronomical Society of the Pacific Conference Series, Vol. 52, Astronomical Data Analysis Software and Systems II, ed. R. J. Hanisch, R. J. V. Brissenden, \& J. Barnes, 173

van Straten, W., Bailes, M., Britton, M., Kulkarni, S. R., Anderson, S. B., Manchester, R. N., \& Sarkissian, J. 2001, Nature, 412,158

Wang, C., Lai, D., \& Han, J. L. 2006, ApJ, 639, 1007

Webbink, R. F. 1984, ApJ, 277, 355

Wegner, G. \& Reid, I. N. 1991, ApJ, 375, 674

York, D. G., et al. 2000, AJ, 120, 1579 